\title{
Modification of Moufarrege Total Posterior Pedicle Mammaplasty: COnical Plicated Central U shaped (COPCU’s) Mammaplasty
}

\author{
Eray Copcu \\ Adnan Menderes University, Medical Faculty, Department of Plastic, \\ Reconstrutive and Aesthetic Surgery, Aydin, Turkey, \\ E-mail: ecopcu@gmail.com \\ Received February 4, 2011; revised March 8, 2011; accepted March 18, 2011
}

\begin{abstract}
Reduction mammaplasty and mastopexy is one of the biggest operation groups which have many techniques and their modifications. Generally accepted that, new modifications are the results of improvements of existing techniques. In this study we present a new modification of Moufarrege total posterior pedicle mammaplasty. We performed central plication to achieve a juvenile look in the superior pole of the breast and to prevent postoperative pseudoptosis and used central U shaped flap to achieve maximum NAC safety and to preserve lactation and nipple sensation. Sixty-nine patients were operated with the above mentioned technique. Out of 69 patients, 52 underwent reduction mammaplasty (11 had gigantomastia), eleven mastopexy, and six oncoplastic. All of the patients were satisfied with functional and aesthetic results and none of them had major complications such as total NAC loss. Only six patients had wound healing problems on the suture line and two patients had minimal hematoma. Since we performed conical plication we would like to evaluate long term effects of the plication in the breast parenchyma. Breast parenchyma was visualized with USG in younger patients and mammography in older patient in postoperative 6 months and 1 year. We never observed any problem related with our sutures and retroareolar part of the areola examination for ductal patency was performed and interestingly all the patients had very clear ductal patency. Our modification is a safe, reliable technique which creates the least scar, avoids previously described disadvantages, provides maximum preservation of functions, can be employed in all breasts regardless of their sizes and is appropriate for oncoplastic surgery and revision surgery.
\end{abstract}

Keywords: Breast Reduction, Mammaplasty, Pedicle

\section{Introduction}

There is no other procedure in breast surgery where the surgeon has a greater opportunity to demonstrate his or her aesthetic abilities than with a breast reduction [1]. There have been numerous studies on reduction mammaplasty and its modifications in the literature. The earliest reports of breast reduction surgery date back hundreds of years. Durston recognized the disability of large female breast in 1670 [2]. The multitude of modifications of reduction mammaplasty indicates that the ideal technique has not yet to be found. There are three reasons for seeking the ideal technique:

One reason is to preserve functional features of the breast: breastfeeding and arousal. Another reason is to achieve the real geometric and aesthetic shape of the breast with the least scar. The last one is to minimize complications of prior surgical techniques without causing an additional complication [3].

In 1970s McKissock introduced the vertical bipedicle breast reduction technique, which was the first procedure for breast reduction surgery that was both reliable and reproducible [4]. Total posterior pedicle was described by Moufarrege [5]. It was called total posterior pedicle since the pedicle was just behind the NAC and the whole posterior pedicle was made of the gland. Total posterior pedicle achieved maximum gland and nipple security and Moufarrege reported low rates of complications in more than 10000 patients undergoing reduction mammaplasty [6]. Moreover, none of them were major complications. 
However, Moufarrege did not recommend total posterior pedicle for very large breasts. It has been recommended that the techniques described so far could be used for reduction of breasts moderate in size. The management of gigantomastia is still debatable. Many authors propose that nipple areola graft can be utilized for the management of gigantomastia. In this study we present a new modification of Moufarrege total posterior pedicle mammaplasty.

We use total posterior pedicle as like in Moufarrege technique; but we create a central located U shaped pedicle for two reasons:

First, we try to prevent post-operative bottoming-out deformity secondary to the bulky tissue of lower part of the breast and, second we create only one space which can be drained only one drain instead of two spaces. We also perform conical plication in the superior part of the breast for juvenile look.

In fact, conical plication creates fullness in the superior pole which in turn leads to an attractive and younger look, the technique does not cause postoperative pseudoptosis which frequently occurs in mammaplasty techniques, central U shaped pedicle allows maximum preservation of functions and the technique is applicable in all breasts irrespective of their sizes. In short, maximum preservation of functions and an aesthetic breast with minimum scar are achieved by Conical Plicated Central U shaped (COPCU’s) mammaplasty.

\section{Material and Methods}

This technique was a modification of the total posterior pedicled mammaplasty described by Moufarrege [6]. The most important feature of the technique was that the central U shaped pedicle was a total posterior pedicle. The "open sky" approach was used and all tissues were easily accessible. Thus, the desirable shape was given and maximum preservation of all anatomical structures was achieved. While central U shaped pedicle was being created, peripheral tissues were resected and posterior and superior connections of the pedicle were preserved completely. The pedicle directly carried the NAC and all vascular and neural connections of the pedicle were preserved.

The first stage of the procedure was marking. A preoperative marking which was quite simple and easy to apply in all patients was developed. As Moufarrege described, the marking was performed when the patient was seated. In order to preserve the axis of each breast crossing the nipple, the vertical axis crossing the nipple and paralleling the margins of the breast was identified and this axis did not have to cross the midclavicular line (Figure 1).
After the axis of the breast was detected, the inframammarian fold was marked. and the upper point of the keyhole pattern was determined. This point was the place where the inframammarian fold was located (Figure 3).

Next, the standard keyhole pattern was marked. Extending arms of the pattern had an angle of 90 degrees and each was $5 \mathrm{~cm}$ in length (Figure 4).

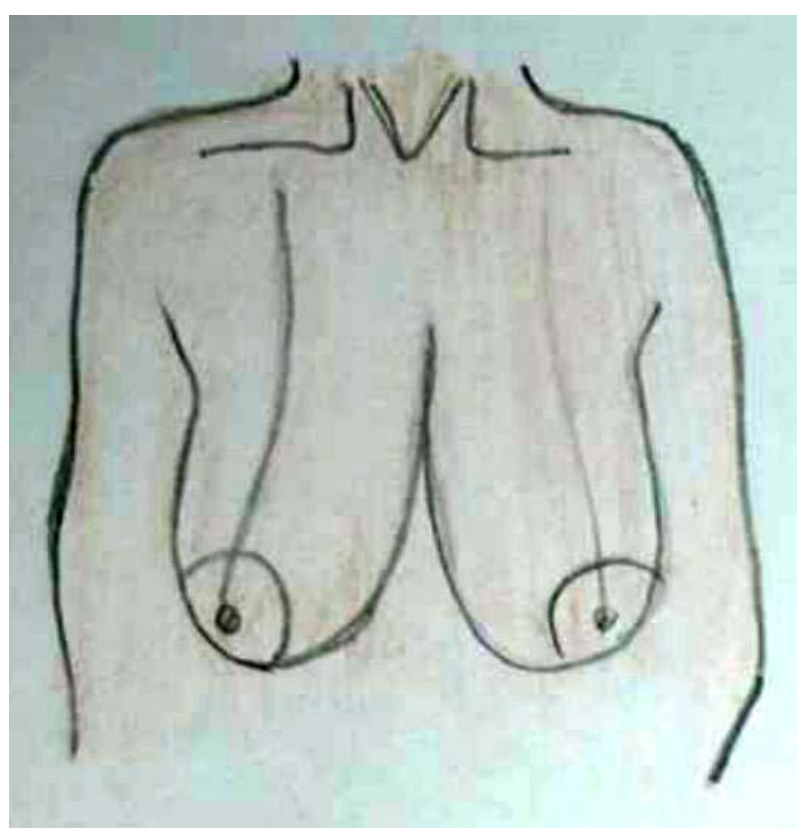

Figure 1. Axis of the breast.

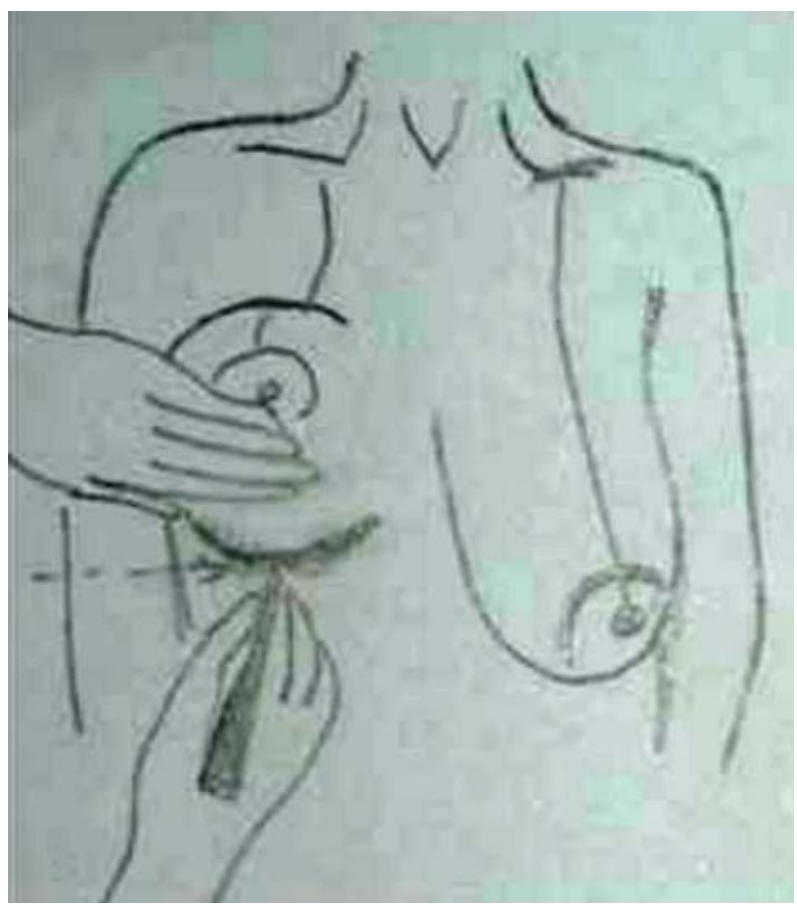

Figure 2. Marking of the infra-mammarian fold. 


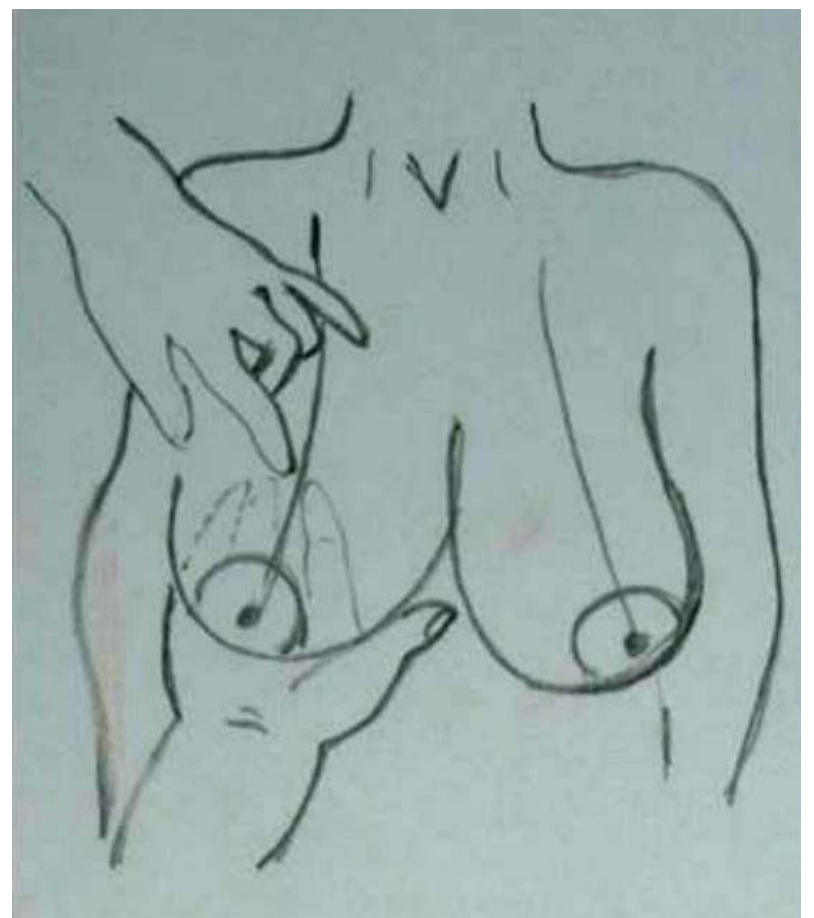

Figure 3. Determining of the upper point of the keyhole pattern.

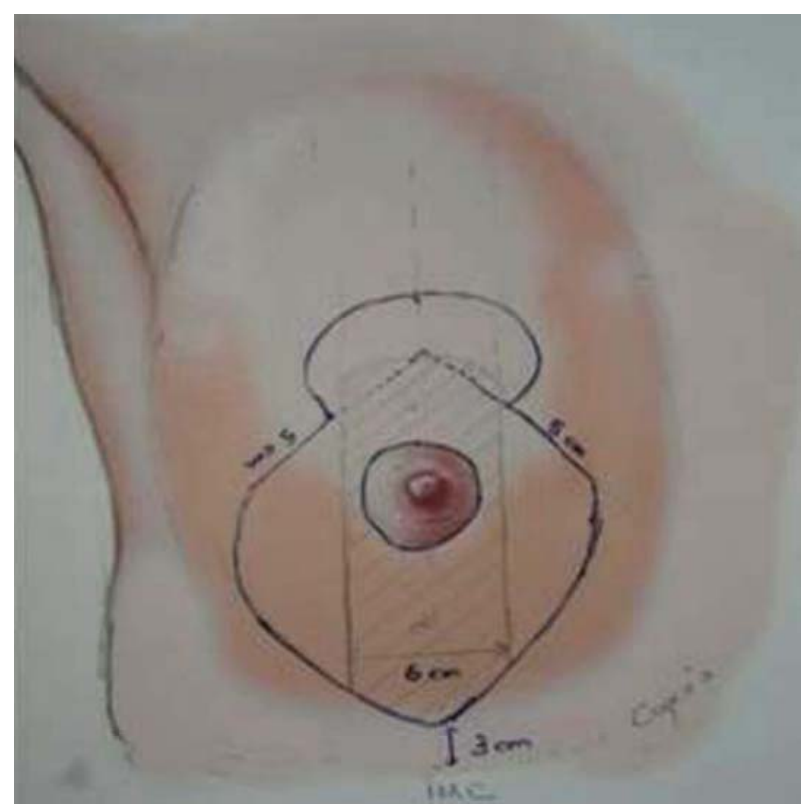

Figure 4. Drawing of the keyhole pattern.

Moufarrege classified breasts into three based on their size when marking the standard keyhole pattern. We increased the angle between the arms of the keyhole to 135 degrees only in cases of gigantomastia. A larger angle is not more advantageous. In fact, creating a larger angle requires harvesting more skin and causes tension on the suture line, which may lead to difficulties in healing.
Arms of the keyhole $5 \mathrm{~cm}$ in length formed a curve $3 \mathrm{~cm}$ above the inframammarian fold (Figure 4). Then, a vertical pedicle $6 \mathrm{~cm}$ in length running the midline of the breast was marked (Figure 5).

It extended to $2 \mathrm{~cm}$ above the NAC in the superior part and till the end of the marked area in the inferior part. Last, the periareolar area $5 \mathrm{~cm}$ in diameter was marked. The second stage was surgery. Patients were in the supine position with a slight flexion in the waist. The tumescent technique was used in all patients. After incisions appropriate for the markings were made, the skin on the pedicle was de-epithelized (Figure 6).

Subsequently, skin flaps were undermined, starting in the medial. The breast including dermal fat was undermined from the gland to aponeurosis of the pectoralis

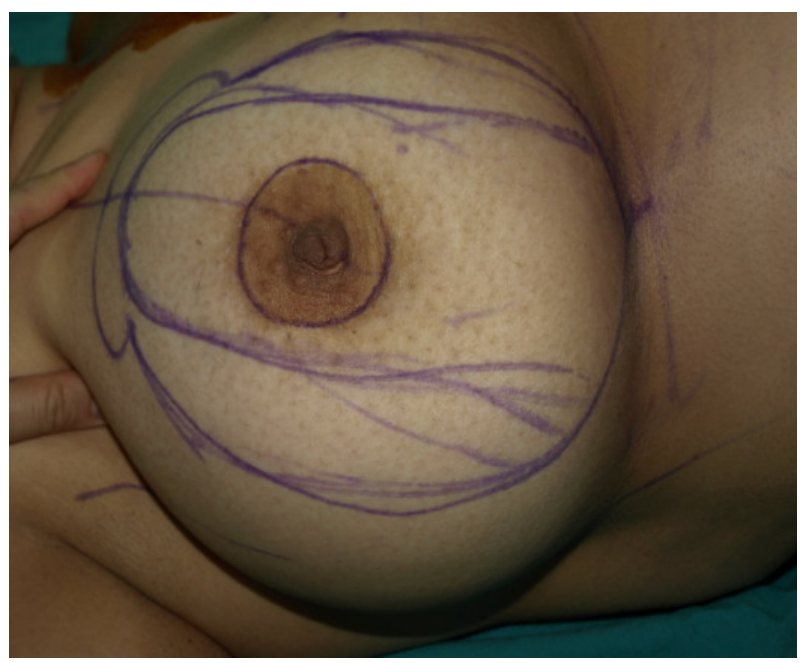

Figure 5. Drawing of the pedicle $6 \mathrm{~cm}$ in length running the midline of the breast.

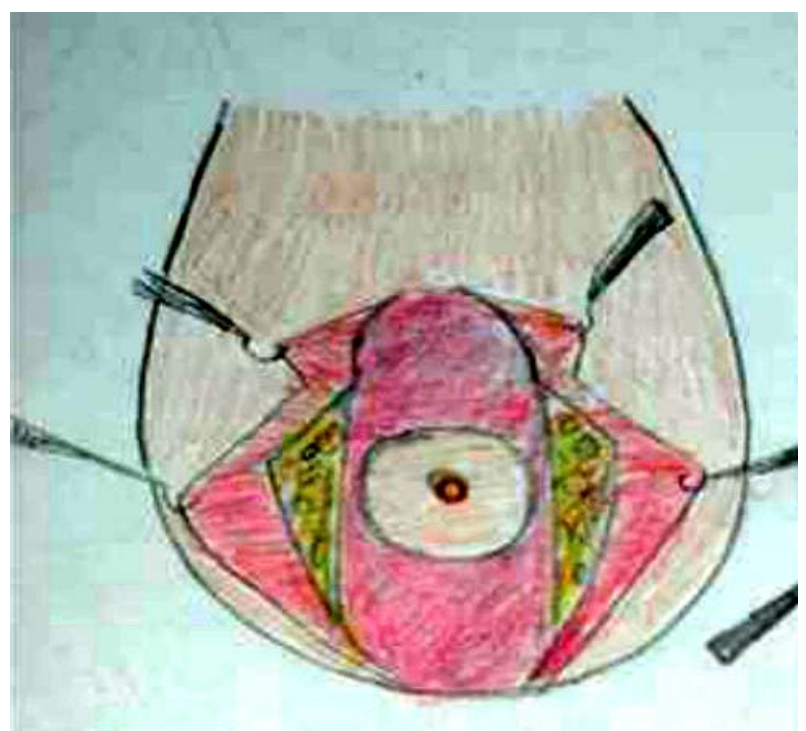

Figure 6. De-epithelisation of the breast. 
major. At the end of undermining, the breast was completely exposed in the front view. Resection of the peripheral tissue started at the medial and continued at the lateral and at the inferior part minimally so as to create a $6 \mathrm{~cm}-\mathrm{U}$ shaped pedicle in the middle. Resection margins in the inferior did not extend beyond the inframammarian fold and no resection was made in the superior. Unlike the posterior pedicle mammaplasty described by Moufarrege, the technique described here involved minimal resection in the inferior, which prevented excess in the horizontal part, and only one hole was created for drainage. Resection of the external quadrant extending to the subaxiallary region was performed gently and the areolar tissue in this area was preserved especially in cases of gigantomastia and extreme hypertrophy (Figure 7).

After the resection was completed, a $U$ shaped total posterior pedicle $6 \mathrm{~cm}$ in width remained in the middle. Following resection, conical plication was carried out to achieve posterior fullness.

Plication was performed in such a way to create a cone at the two o'clock and ten o'clock positions of the NAC with oblique continuous suture with 2/0 PDS (Figure 8).

After conical plication was created, the breast was secured in its new position with temporary sutures running

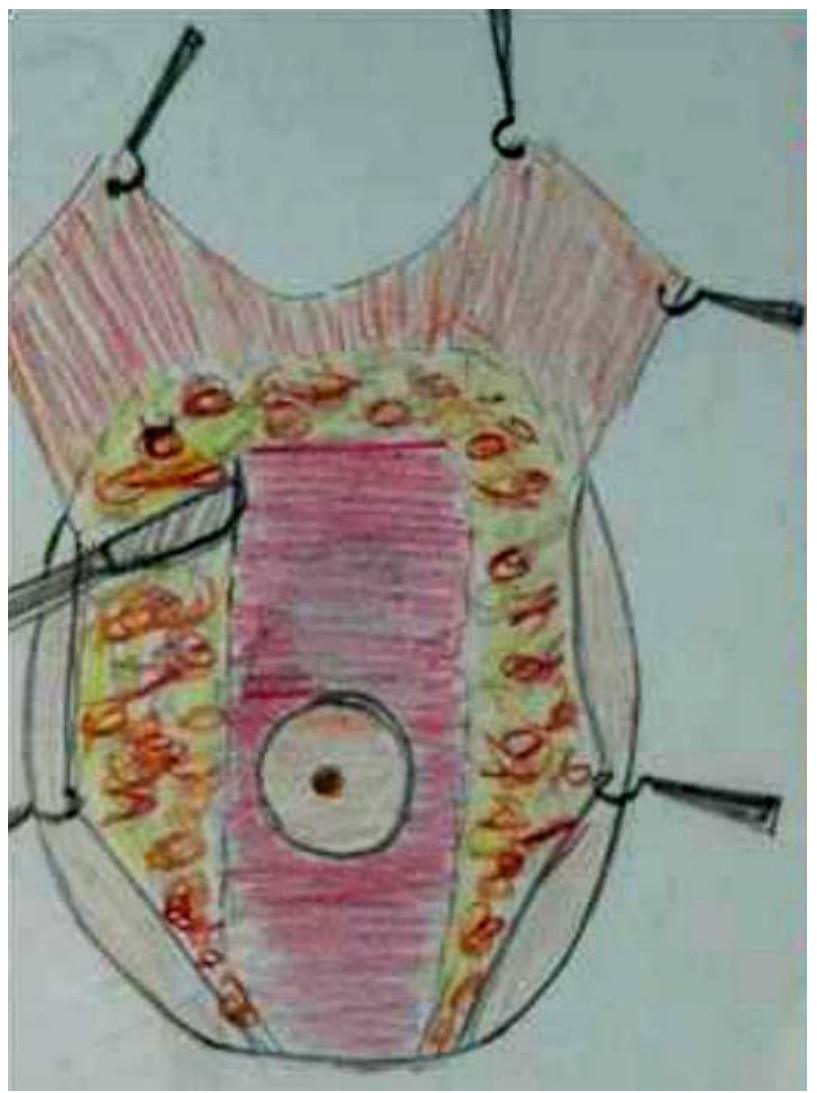

Figure 7. U shaped total posterior pedicle $6 \mathrm{~cm}$ in width.

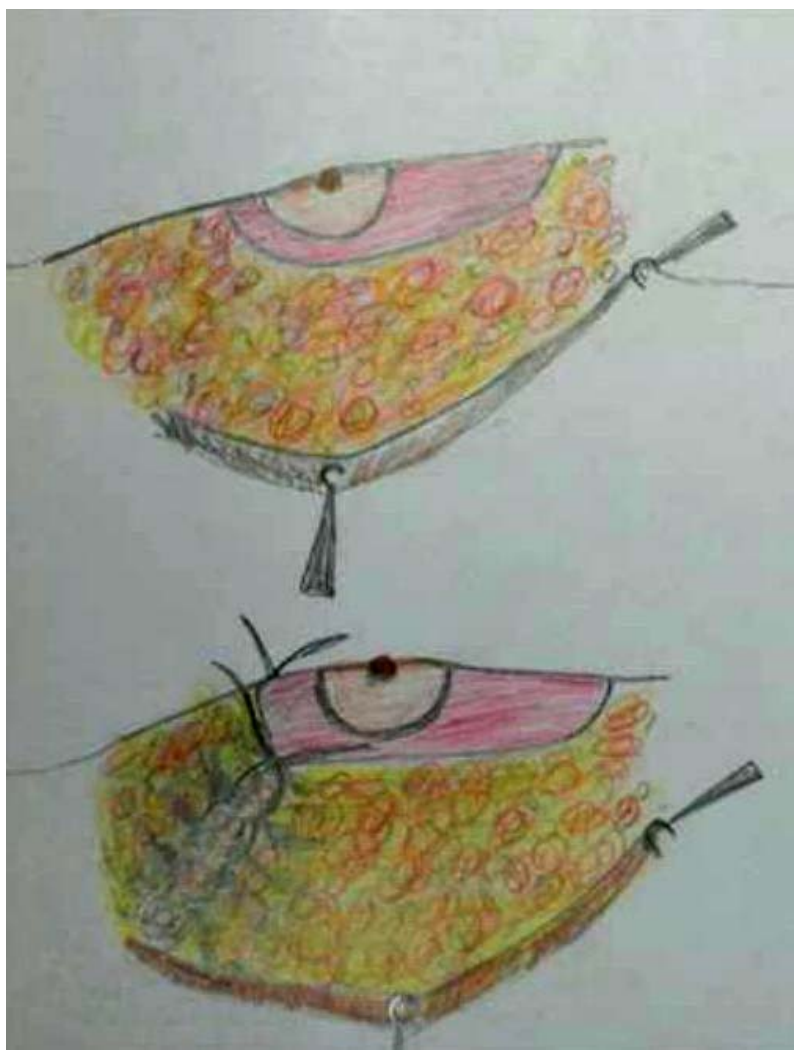

Figure 8. Conical plication of the pedicle.

through inferior and superior parts of the NAC. One vertical suture was put $6 \mathrm{~cm}$ below the NAC and the area below this point was closed with pursing sutures. The subdermis was closed with 3/0 PDS, the vertical incision with 4/0 PDS and the periareolar region with 5/0 PDS without tension. One drainage tube (Hemovac ${ }^{\circledR}$ ) was placed and temporary sutures were removed at the end of the operation. Only a short vertical scar appeared in all cases and reverse $\mathrm{T}$ incision was avoided. Pressure dressing was done at the end of the operation and the drain tubes were removed within two days of the operations.

A detailed physical examination of the breast includes measurements of breast size, degree of ptosis, masses, superior pole fullness, nipple sternal distance, nippleinframmammary fold distance were recorded. SemmesWeinstein monofilaments were used to test the sensitivity of the nipple and cardinal points of the areola before surgery and 3, 6, and 12 months after surgery (Figure 9).

\section{Results}

COPCU's mammaplasty was performed in 69 patients. The median age of the patients was 24,2 years, ranging from 17 years to 66 years. Data of the patients are presented in Table: 


\begin{tabular}{lll}
\hline & Range & Average \\
\hline Age & $17-66$ & 24.2 \\
$\begin{array}{l}\text { Jugular notch to nipple distance } \\
\text { Pre-operative }\end{array}$ & $21-43 \mathrm{~cm}$. & $27 \mathrm{~cm}$. \\
$\begin{array}{l}\text { Post-operative } \\
\text { Nipple to inframammarian crease }\end{array}$ & $18-24 \mathrm{~cm}$. & $21 \mathrm{~cm}$. \\
$\begin{array}{l}\text { Pre-operative } \\
\text { Post-operative }\end{array}$ & $8-18 \mathrm{~cm}$. & $14.5 \mathrm{~cm}$. \\
Resection weight(per breast) & $7-12 \mathrm{~cm}$. & $9 \mathrm{~cm}$. \\
Follow up & $110-1880 \mathrm{gr}$ & $480 \mathrm{gr}$ \\
\hline
\end{tabular}
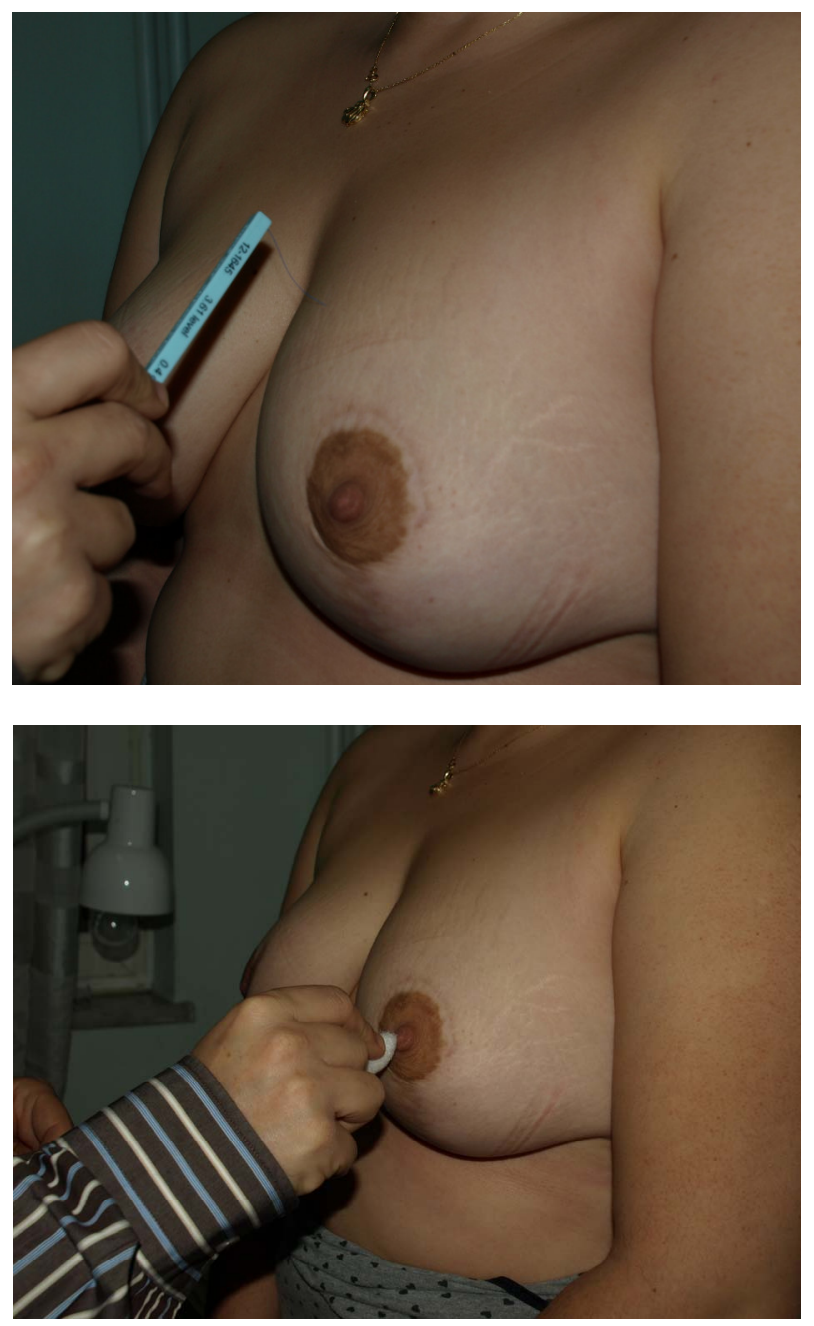

Figure 9. Evaluation of the sensitivity of the breast with Semmes-Weinstein monofilaments and control of the erection of nipple with “cotton test”.

The mean distance between the xiphoid process and the areola was $27 \mathrm{~cm}$, ranging between $21 \mathrm{~cm}$ and $43 \mathrm{~cm}$. The mean resected tissue weight per breast was $480 \mathrm{gr}$, ranging from 110 gr to $1880 \mathrm{gr}$. Out of 69 patients in- cluded in the study, 52 underwent reduction mammaplasty (11 had gigantomastia), eleven mastopexy, and six oncoplastic surgery.

Out of six patients undergoing oncoplastic surgery, two underwent COPCU's mammaplasty and the superior part where the tumors were located was resected. Both patients underwent breast reduction. In the remaining four patients undergoing oncoplastic surgery, partial mastectomy was performed based on the markings described in COPCU's mammaplasty, breast prostheses were placed under pectoralis muscle and the opposite breast was reduced.

The patients were followed for six months minimum. Lateral photographs were taken to compare preoperative and postoperative superior fullness in all patients (Figure 10-13).

None of the patients had such total NAC necrosis. Partial NAC loss (approximately 10\% of the NAC) was seen in one patient who was heavy smoker and she did not stop smoking even our advice. Necrotic areas were healed spontaneously with routine dressings without any surgical approach. Two days after removal of the drainage tubes, two patients had minimal hematoma, which was treated conventionally. Two patients had about $3 \mathrm{~cm}$ opening on the NAC and suture line, but they healed

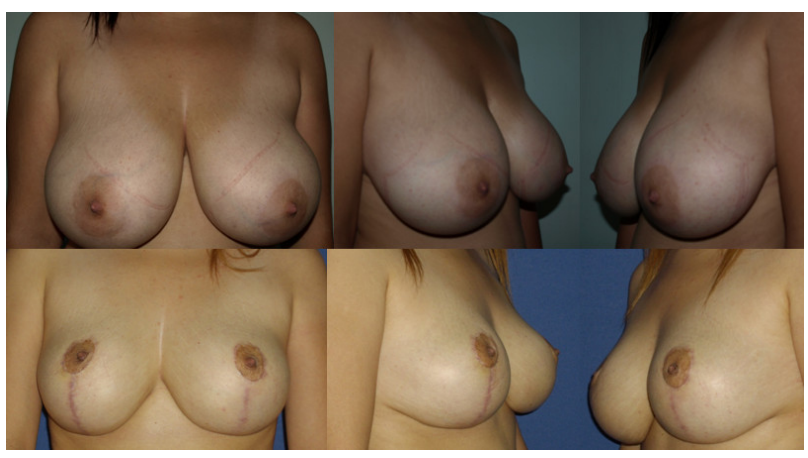

Figure 10. Pre and post-operative view of the patient operated with COPCU's mammaplasty for reduction of the breast.

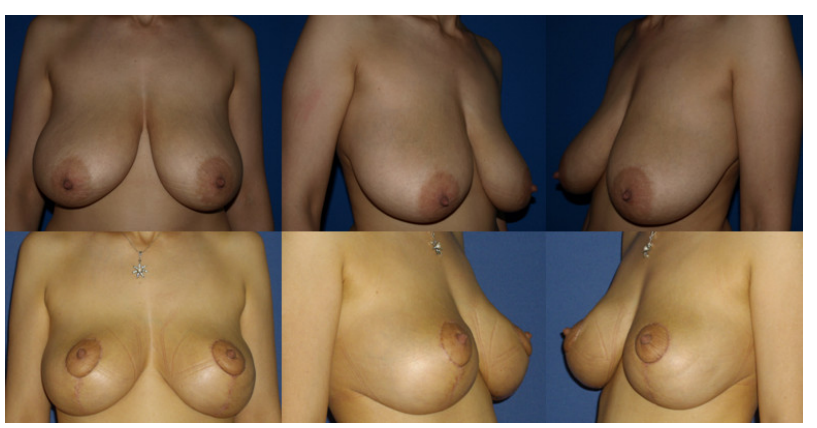

Figure 11. Pre and post-operative views of the patient operated with COPCU's mammaplasty for reduction of the breast. 


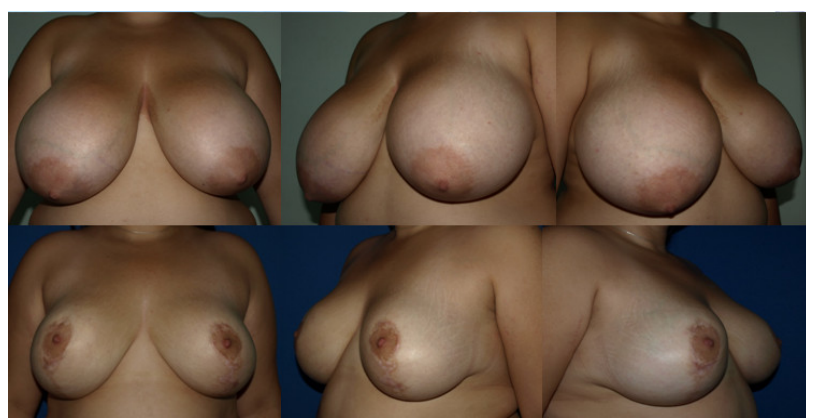

Figure 12. Pre and post-operative view of the patient operated with COPCU's mammaplasty for reduction of the breast.

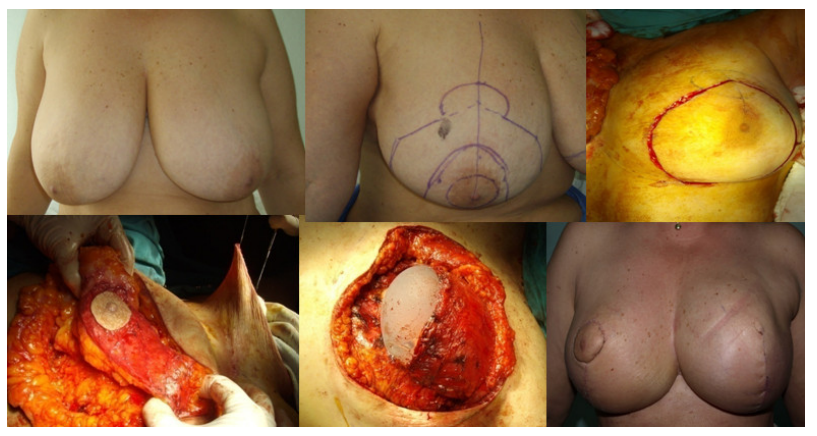

Figure 13. Pre and post-operative view of the patient with breast Ca. $350 \mathrm{ml}$. Baker prosthesis was applied for the left breast.

spontaneously. Four patients were operated for scar revision surgery under the local anesthesia. All patients were satisfied with aesthetic results. None of the patients reported decreased sensual or sexual sensibility in the short term and long term. Six patients gave birth within six months of the operations and none of them had decreased lactation. Since we performed conical plication we would like to evaluate long term effects of the plication in the breast parenchyma. Breast parenchyma was visualized with USG in younger patients and mammography in older patient in postoperative 6 months and 1 year. We never observed any problem related with our sutures and retroareolar part of the areola examination for ductal patency was performed and interestingly all the patients had very clear ductal patency (Figure 14).

\section{Discussion}

The goal of aesthetic surgery is generally accepted to be to re-shape normal structures of the body to improve patient's appearance and self esteem [7]. The surgeons who perform breast reduction surgery or mastopexy have great responsibility because of the breast is exceptional organ. The breast is one of the most important organs of women. It plays a role not in sexuality due to its visual

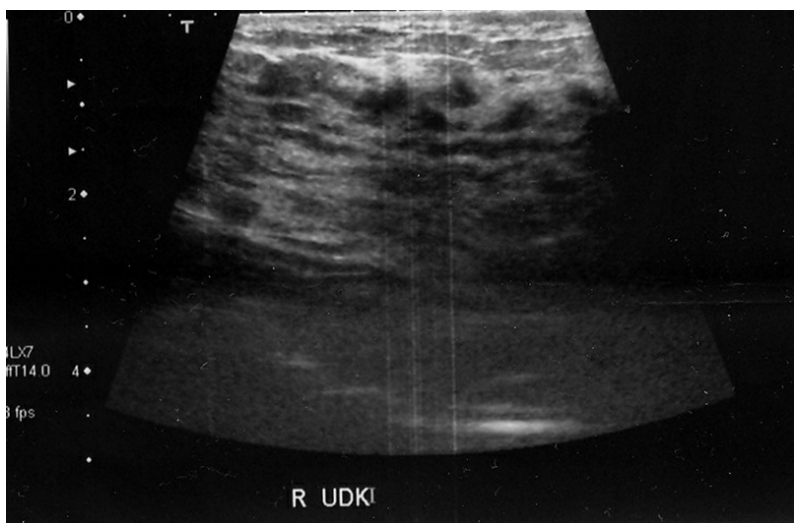

Figure 14. Ultrasonography of the patient operated with COPCU's mammaplasty.

and sensual importance but also in reproduction due to its capability of milk production. None of the plastic surgery operations put as much a heavy burden on plastic surgeons as reduction mammaplasty [3].

Reduction mammaplasty techniques described so far are named after locations of pedicles. Among them are inferior, lateral, medial, central, total posterior pedicle and mixed [8-11].

The leading cause of ongoing attempts to seek an ideal technique is complications such as failure to achieve the desirable aesthetic result, decreased or lack of lactation, decreased or loss of sensual and erogenous feeling of the nipple, insufficient projection and postoperative pseudoptosis and wound healing problems. Ultimate goal of any pedicle is to provide sufficient blood supply to the nipple areola complex [12]. It has been reported that superior pedicled mammaplasty causes considerable changes in blood circulation due to the transposition of the pedicle and that there is decreased NAC sensation in the superior pedicle in the short term. The nerves innervate the NAC can be easily injured with inferior pole resections with superior pedicle techniques [13,14]. Bottoming out, inferior pole excess or pseudo ptosis is more frequent in inferior based pedicles [14]. Attempts to seek reduction mammaplasty techniques preserving the NAC emerged from the results of the studies by Bisenberger [15]. However, they revealed considerably high rates of complications. Moufarrege published his own technique in 1985 as "Total Dermoglandular Pedicle" and also he presented his largest series in 2006 with more than 5000 patients $[5,6]$. Moufarrege has used $100 \%$ of the remaining gland for the nipple-areola complex. The pedicle initially was in posterior and inferior position, but eventually it occupied the entire height of the gland. This was the total posterior pedicle [6].

Nipple necrosis is the most frightening complication of reduction mammaplasty. The rates of nipple necrosis have been reported to be $2.1 \%$ in the superodermal pedi- 
cle [16], 2.3\% in the superolateral [17] and $0.8 \%$ in inferior pedicle [18]. The leading cause of nipple necrosis is insufficient arterial blood supply or long-lasting venous congestion; this can be attributed to inadequate knowledge about the vascular anatomy of the NAC and use of long peripheral pedicle and the resultant distortion of the pedicle. However, total posterior pedicle described by Moufarrege and its modification COPCU's mammaplasty have theoretically avoided such complications.

Functional results of breast reduction are as important as its aesthetic results. The Surgeon General's health goals for 2010 are that $75 \%$ of women initiate breastfeeding and that $50 \%$ continue it through 6 months postpartum [19]. Maximum preservation of breast functions depends on exact knowledge of anatomical features of the breast. At present, vascularization and innervations of the nipple areola complex (NAC) has been clearly described and the vessels and the nerves have been shown to reach vertically the NAC at the fourth and fifth ribs through a separate fibrous septum [20,21]. It should be noted that vascularization and innervation of the NAC is through the central breast parenchyma which can be seen as inferior to the breast shape in standing position. If the glandular tissue is not removed with the central pedicle, then the patient keeps her lactation potential with good nipple sensation [22]. The principle underlying the technique described here is complete preservation of these tissues. Any technique which avoids resection of the central parenchyma does not detach the central part of the breast from thoracic wall and does not violate the lateral pectoral fascia carries a low risk of injuring the dominant nerve supply to the nipple and areola [23].

A larger pedicle does not necessarily achieve better breast functions. The thing is that vessels and nerves of the NAC should be completely preserved. As a matter of fact, a large pedicle may cause such complications as displacement and folding of the flaps [24]. So that the breast looks natural after reduction mammaplasty, it can move to all directions and has a soft texture. This can only be achieved with a total posterior pedicle since it is not possible for a flap from the neighboring areas to achieve natural mobility of the breast.

It has been emphasized that a gland connected to the ducts and the nipple should be preserved for a successful breastfeeding following breast reduction [25]. However, to our knowledge, there have not been any studies showing how much breast tissue should be preserved for sufficient milk production. Maintenance of lactation should never be disregarded. Therefore, a maximum amount of the gland should be preserved. Only a pedicle located on the gland allows preservation of a maximum amount of the gland, which was only achieved by Moufarrege total posterior pedicle mammaplasty and its modifications.
In our technique we perform three important modifications to the original technique of the Moufarrege. First, we create central located U shaped total posterior pedicle. The philosophy of the pedicle is as same as Moufarrege technique and pedicle is $100 \%$ of remaining gland. But we excise some breast tissue from the lower part of the breast. This allows using only one drain since we create single space instead of two and more importantly we prevent the possibility of the bottoming-out deformity in post-operative period especially in gigantomastia.

Secondly, we describe the term of conical plication in the breast. As far as we know, conical plication has not been described in the literature before. The conical plication which we developed is directed towards preservation of the juvenile breast look and superior fullness in the long term.

In 1985, Pennington performed plication and pedicle suspension in the pectoral fascia to prevent bottoming out, a frequently encountered complication of inferior pedicle, and reported his 20-year experience [26]. Pennington made plication, both superficial and deep, in the inferior pole. Unlike the plication by Pennington, plication in COPCU's mammaplasty is performed in the superior only to create a conical appearance. The suture technique used in COPCU's mammaplasty is similar to that described by Tonnard for MACS lift [27]. However, the technique presented here does not damage the tissue since it only involves plication and no problems due to plication were shown in postoperative mammography in the long term. The size of the breast was not associated with complications in the present series. This can be ascribed to safety of the pedicle.

There is no limitation of the breast size for COPCU's mammoplasty; even it can be used for the correction of the gigantomastia with success (Figure 15)

Finally, we use vertical skin closure instead of inverted $\mathrm{T}$ scar and prevent the possibility of hypertrophic scars or keloid on horizontal part of the scar.

According to the results of this study and the studies of Moufarrege, advantages of total posterior pedicle mammaplasty and its modification can be listed as in the following [6]:

1) Since it is a total posterior pedicle, it provides complete safety of the NAC. The nipple is located on the pedicle and the vessels and nerves extending the nipple are completely protected. In fact, we did not observe any complications concerning the nipple.

2) The pedicle is in the midline and involves the whole gland. Therefore, it is one of the best to create the most natural breast in terms of tissue consistency and mobility.

3) A young breast has fullness in the superior. Conical plication does not only create a juvenile look of the breast but also prevents such complications of classic 

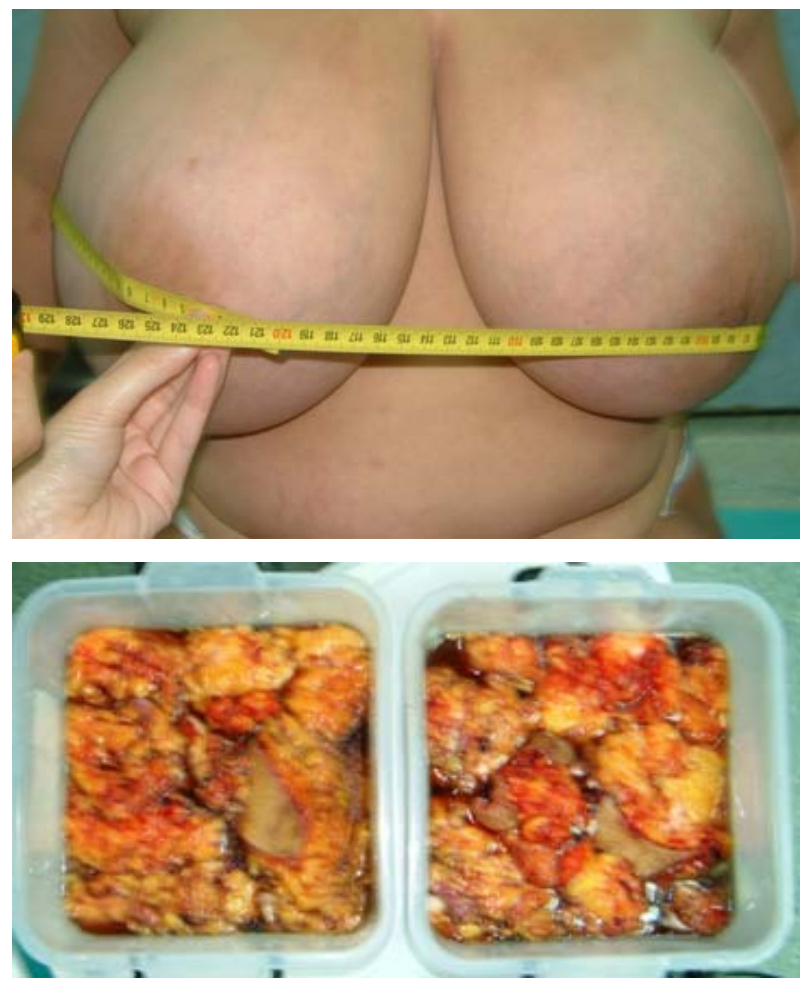

Figure 15. Patient with gigantomastia and excised materials from breasts.

mammaplasty as loosening of the suture put on the pectoral muscle, flattening in the superior pole and pseudoptosis.

4) Moufarrege total posterior pedicle mammaplasty offers excellent projection of the breast. In fact, the whole pedicle is made of the gland and located in the central, which helps to achieve a near normal projection.

5) The technique provides maximum protection of the sensual and sexual innervation of the NAC.

6) It is one of safest techniques for lactation since the pedicle is situated on the gland.

7) It is easy to perform and teach since open sky approach is used. It does not increase operation time and does not require liposuction.

8) External quadrants of the breast, most susceptible to cancer, were resected. This is a kind of prophylaxis against cancer.

9) Reverse T scar is avoided and a very small vertical scar, which can be tolerated by patients, is created.

There are not any marked disadvantages of the technique. However, thinning likely to occur in elevation of dermal pedicles may cause skin problems. Although the patients included in this study were heavy smokers, they did not have skin loss. This indicates that dermal flaps have a rich blood supply.

It can be concluded that Moufarrege total posterior pedicle and it's modification COPCU's mammaplasty cannot only be used safely for breast reduction and mastopexy, in which a young projectile breast is created and maximum protection of breast functions is provided, but also is appropriate for oncoplastic surgery.

\section{References}

[1] D. C. Hammond. "Breast Reduction”, Atlas of Aesthetic Breast Surgery, 2009, p. 146.

[2] A. Berg, B. Stark E. and Malec, "Reduction Mammaplasty: A Way Helping Females with Neck, Shoulder and Back Pain Symptoms," European Journal of Plastic Surgery, Vol. 17, No. 2, 1994, pp. 84- 86.

[3] E.Copcu, “A Versatile Breast Reduction Technique: Conical Plicated Central U Shaped (Copcus) Mammaplasty," Annals of Surgical Innovation and Research, Vol. 3, No. 7, 2009.

[4] E. J. Hall-Findlay, "Pedicles in Vertical Breast Reduction and Mastopexy," Clinic Plastic Surgery, Vol. 29, No. 3, 2002, pp. 379-391.doi:10.1016/S0094-1298(02)00008-1

[5] R. Moufarrege, G. Beauregard, J. P. Bosse, G. Muller, J. Papillon and C. Perras, "Reduction Mammoplasty by the Total Dermoglandular Pedicle,” Aesthetic Plastic Surgery, Vol. 9, No. 3, 1985, pp. 227-232. doi:10.1007/BF01570855

[6] R. Moufarrege, 2006, http://www.emedicine.com/plastic/ topic488.htm

[7] M. C. Ferreira, "Evaluation of Results in Aesthetic Plastic Surgery: Preliminary Observations on Mammaplasty," Plastic and Reconstructive Surgery, Vol. 106, No. 7, 2000, pp. 1630-1635. doi:10.1097/00006534-200012000-00032

[8] A. Ceydeli, J. Louis, J. Yu and E. Ritter, "Lateral Septal Preservation: a Technique to Improve Projection in Inferior Pedicle Reduction Mammaplasty by Preventing Lateral Displacement of the Pedicle," Plastic and Reconstructive Surgery, Vol. 120, No. 4, 2007, pp. 1088-1089. doi:10.1097/01.prs.0000278185.71693.18

[9] W. Hefter, P. Lindholm and O. P. Elvenes, "Lactation and Breast-Feeding Ability Following Lateral Pedicle Mammaplasty,” British Journal of Plastic Surgery, Vol. 56, No. 8, 2003, pp. 746-751. doi:10.1016/S0007-1226(03)00368-0

[10] M. Y. Nahabedian, B. M. McGibbon and P. N. Manson, "Medial Pedicle Reduction Mammaplasty for Severe Mammary Hypertrophy," Plastic and Reconstructive Surgery, Vol. 105, No.3, 2000, pp. 896-904. doi:10.1097/00006534-200003000-00011

[11] T. R. Hester and J. Cukic, "Central Breast Pedicle and 'Free-Hand' Technique for Alteration of Volume and Skin Envelope of the Breast," Clinic Plastic Surgery, Vol. 15, No. 4, 1988, pp. 613-625.

[12] P. Andrades and A. Prado, "Understanding Modern Breast Reduction Techniques with a Simplified Approach,” Journal of Plastic, Reconstructive \& Aesthetic Surgery, Vol. 61, No. 11, 2008, pp. 1284-1293. doi:10.1016/j.bjps.2007.11.060 
[13] I. Schlenz, S. Rigel, M. Schemper and R. Kuzbari, “Alteration of Nipple and Areola Sensitivity by Reduction Mammaplasty: a Prospective Comparison of Five Techniques,” Plastic and Reconstructive Surgery, Vol. 115, No. 3, 2005, pp. 743-751. doi:10.1097/01.PRS.0000152435.03538.43

[14] S. O. Harbo, E. Jorum, H. E. Roald, "Reduction Mammaplasty: a Prospective Study of Symptom Relief and Alterations of Skin Sensibility," Plastic and Reconstructive Surgery, Vol. 111, No. 1, 2003, pp. 103-110. doi:10.1097/00006534-200301000-00017

[15] H. Biesenberger, "Ein Neue Methode Der Mammoplastik Zentralbl Chir,” Vol. 55, No. 4, 1928, p. 2382.

[16] P. N. Blondeel, M. Hamdi, K. A. Sijpe Van de, K. H. Van Landuyt, F. E. Thiessen and S. J. Monstrey, "The Latero-Central Glandular Pedicle Technique for Breast Reduction,” British Journal of Plastic Surgery, Vol. 56, No. 4, 2003 348-359. doi:10.1016/S0007-1226(03)00191-7

[17] L. Cardenas-Camarena and R. Vergara, "Reduction Mammaplasty with Superior-Lateral Dermoglandular Pedicle: Another Alternative," Plastic and Reconstructive Surgery, Vol. 107, No.3, 2001, pp. 693-699. doi:10.1097/00006534-200103000-00007

[18] A. D. Mandrekas, G. J. Zambacos and A. Anastasopoulos, "Hapsas DA. Reduction Mammaplasty with the Inferior Pedicle Technique: Early and Late Complications in 371 Patients,” British Journal of Plastic Surgery, Vol. 49, No. 7, 1996, pp. 442-446. doi:10.1016/S0007-1226(96)90027-2

[19] U.S. Department of Health and Human Services, Healthy People, 2010.

[20] H. Nakajima, N. Imanishi, S. Aiso, “Arterial Anatomy of the Nipple-Areola Complex,” Plastic and Reconstructive Surgery, Vol. 96, No. 4, 1995, pp. 843-845. doi:10.1097/00006534-199509001-00012

[21] E. Wuringer, N. Mader, E. Posch and J. Holle, "Nerve and Vessel Supplying Ligamentous Suspension of the Mammary Gland,” Plastic and Reconstructive Surgery, Vol. 101, No. 6, 1998, pp. 1486-1493. doi:10.1097/00006534-199805000-00009

[22] M. Keskin, Z. Tosun and N. Savaci, "Seventeen Years of Experience with Reduction Mammaplasty Avoiding a Vertical Scar,” Aesthetic Plastic Surgery, Vol. 32, No. 4, 2008, pp. 653-659. doi:10.1007/s00266-008-9167-1

[23] R. Kuzbari and I. Schlenz, "Reduction Mammaplasty and Sensitivity of the Nipple-Areola Complex: Sensuality Versus Sexuality?” Ann Plast Surg, Vol. 58, No. 1, 2007, pp. 3-11. doi:10.1097/01.sap.0000250648.70177.4a

[24] D. L. Abramson, S. Pap, S. Shifteh and S. B. Glasberg, "Improving Long-Term Breast Shape with the Medial Pedicle Wise Pattern Breast Reduction,” Plastic and Reconstructive Surgery, Vol. 115, No. 7, 2005, pp. 19371943. doi:10.1097/01.PRS.0000165085.23537.A7

[25] N. I. Cruz and L. Korchin, "Lactational Performance after Breast Reduction with Different Pedicles,” Plastic and Reconstructive Surgery, Vol. 120, No. 1, 2007, pp. 35-40. doi:10.1097/01.prs.0000263371.37596.49

[26] D. G. Pennington, "Improving the Results of Inferior Pedicle Breast Reduction Using Pedicle Suspension and Plication,” Aesthetic Plastic Surgery, Vol. 30, No. 4, 2006, pp. 390-394. doi:10.1007/s00266-006-0040-9

[27] P. L. Tonnard, A. Verpaele and S. Gaia, "Optimising Results from Minimal Access Cranial Suspension Lifting (MACS-lift)," Aesthetic Plastic Surgery, Vol. 29, No. 4, 2005, pp. 213-220. doi:10.1007/s00266-005-0047-7 